\title{
ORIGINAL ARTICLE \\ Residual urine volumes after intermittent catheterization in men with spinal cord injury
}

\author{
J Krebs ${ }^{1}$, P Bartel ${ }^{2}$ and J Pannek ${ }^{2}$
}

Study design: Prospective cross-sectional study.

Objectives: To investigate residual urine volumes after intermittent catheterization (IC) in men with spinal cord injury (SCI) and the effect of residual urine on the rate of symptomatic urinary tract infections (UTIs).

Setting: Single $\mathrm{SCl}$ rehabilitation center in Switzerland.

Methods: Sixty men experienced in IC ( $\geqslant 6$ months) emptied their bladder twice by IC. Immediately after catheterization, residual urine was determined by ultrasonography. Personal characteristics and bladder diary details (annual UTI rate, catheter type) were also collected.

Results: The median residual urine volume was $7.0 \mathrm{ml}$ (lower quartile (LQ): 0.0, upper quartile (UQ): $20.3 \mathrm{ml}$ ). No residual urine was observed after $42 \%(n=50)$ of all catheterizations $(n=120)$. Unsatisfactory residual volumes (that is, $>50 \mathrm{ml})$ were observed after $9 \%(n=11)$ of all catheterizations. There was no significant $(P=0.95)$ difference between the median residual urine volume of men with recurrent ( $>2$ UTIs per year) UTIs $(2.5 \mathrm{ml}, \mathrm{LQ}: 0.0, \mathrm{UQ}: 29.3 \mathrm{ml}$ ) and the volume of those with sporadic ( $\leqslant 2$ UTIs per year) UTIs (6.0 ml, LQ: 0.0, UQ: $20.0 \mathrm{ml}$ ).

Conclusions: Bladder evacuation by IC is an efficient method, resulting in zero or small residual urine volumes. The small residual urine volumes generally observed after IC do not predispose for UTIs.

Spinal Cord (2013) 51, 776-779; doi:10.1038/sc.2013.81; published online 13 August 2013

Keywords: residual urine; intermittent catheterization; urinary tract infection

\section{INTRODUCTION}

Pioneered by Guttmann and Frankel ${ }^{1}$ and Lapides et al., ${ }^{2}$ intermittent catheterization (IC) is now the standard method for bladder evacuation in spinal cord injury (SCI) patients suffering from urinary retention as a result of neurogenic lower urinary tract dysfunction (NLUTD). ${ }^{3}$ However, there are only sparse data on the efficiency of IC in evacuating urine from the bladder, indicating that there is some residual urine after IC in certain patients. ${ }^{4-6}$

Residual urine is considered to be an important risk factor for urinary tract infections (UTIs), ${ }^{7,8}$ and even small volumes may predispose for infections. ${ }^{4}$ Although less frequent than with indwelling catheterization, ${ }^{9,10}$ recurrent UTIs are a major problem affecting SCI patients with NLUTD using IC, even when applying adequate catheterization techniques. ${ }^{11}$ Merritt et al..$^{8}$ have reported greater residual urine volumes after IC in SCI patients with bacteriuria compared with those with negative urine cultures. However, there are no current data on residual urine volumes after IC in SCI patients and their effect on the occurrence of symptomatic UTIs.

We have therefore prospectively investigated the residual urine volumes after IC in men with NLUTD resulting from SCI. In addition, the hypothesis that individuals with recurrent ( $>2$ UTIs per year) symptomatic UTIs show greater residual urine volumes after IC compared with those with sporadic ( $\leqslant 2$ UTIs per year) symptomatic UTIs was tested.

\section{MATERIALS AND METHODS}

The present prospective study had been approved by the local ethics committee, and all study participants had provided written informed consent with guarantees of confidentiality. All applicable institutional and governmental regulations concerning the ethical use of human volunteers were followed during the course of this research. The study protocol had been registered with ClinicalTrials.gov (NCT01601041).

Men with NLUTD resulting from SCI using IC for bladder evacuation and presenting for a urodynamic examination at the outpatient clinic of a single SCI rehabilitation center between December 2011 and January 2013 were recruited for the study. Individuals younger than 18 years, inexperienced in IC (IC for $<6$ months) or suffering from a current symptomatic UTIs were excluded. For evaluating the effects of residual urine on the rate of symptomatic UTIs, additional exclusion criteria were applied: age $>65$ years, antibiotic treatment $>28$ days within the previous 12 months and medical conditions detrimental to the immune response.

The participating men emptied their bladder twice (before and after urodynamic examination with maximum bladder filling) by IC according to their standard procedure. Immediately after catheterization, residual urine was determined by the same investigator (JP) using ultrasonography (General Electrics, Glattbrugg, Switzerland) with the participants in the supine position. The intra-observer reliability was 0.90 (unpublished data). The residual urine volume was calculated as follows: $\pi / 6 \times$ sagittal height $\times$ sagittal depth $\times$ transverse width. ${ }^{12}$ As defined previously, a volume of $\leqslant 50 \mathrm{ml}$ was considered to be satisfactory. ${ }^{4}$

Personal characteristics, type of NLUTD, medication and bladder diary details were collected. The study participants were asked to report the number of symptomatic UTIs they have experienced during the last 12 months.

${ }^{1}$ Clinical Trial Unit, Swiss Paraplegic Centre, Nottwil, Switzerland and 2Department of Neurourology, Swiss Paraplegic Centre, Nottwil, Switzerland Correspondence: Professor J Pannek, Department of Neurourology, Swiss Paraplegic Centre, Guido A. Zäch Strasse 1, Nottwil CH-6207, Switzerland. E-mail: juergen.pannek@paraplegie.ch

Received 22 April 2013; revised 25 June 2013; accepted 27 June 2013; published online 13 August 2013 
A symptomatic UTI was defined according to published criteria: fever without another cause, dysuria, new onset of incontinence, suprapubic pain, impaired general well-being, decreased bladder capacity, increased general spasticity, hematuria without other causes and new onset of voiding problems. ${ }^{7,13}$ According to the reported number of symptomatic UTIs during the last 12 months, study participants were categorized as suffering from recurrent ( $>2$ UTIs per year) or sporadic ( $\leqslant 2$ UTIs per year) UTIs. ${ }^{14}$

Data were calculated and presented as the median and the lower quartile (LQ) and upper quartile (UQ). The differences between groups were tested with the Wilcoxon rank-sum and the Kruskal-Wallis test. The $\chi^{2}$-test and Fisher's exact test were used to test differences in the observed frequency distribution between groups. The Spearman rank-order analysis was used to investigate correlations between variables. Statistical analyses were performed using the SPSS software (version 18.0.3, IBM, Somers, NY, USA). A P-value of $<0.05$ was considered significant.

\section{RESULTS}

Residual urine volumes after IC

The data of 60 men with NLUTD resulting from SCI (median 13.8 years, LQ: 5.9, UQ: 21.0 years post injury) and a median age of 47.9 years (LQ: 39.2, UQ: 57.2 years), using IC for bladder evacuation since 9.8 years (median, LQ: 4.4; UQ: 13.4 years), were analyzed. No participants were excluded from the analysis of residual urine volumes (Table 1). Trauma was the main etiology of SCI $(n=51,85.0 \%)$. In four and one men, SCI had resulted from tumorous and inflammatory processes, respectively. Furthermore, there were four men with myelomeningocele. The majority of participants $(n=51,85.0 \%)$ suffered from suprasacral lesions. There were six tetra- (C4-C7) and 54 paraplegic (T3-S4) men. All tetraplegic men were able to aptly perform IC. SCI was complete (American Spinal Injury Association

Table 1 Residual urine volumes after intermittent catheterization in men with spinal cord injury

$\begin{array}{ccc}1 \text { st } & \text { 2nd } & \text { Total } \\ \text { catheterization } & \text { catheterization } & \mathrm{n}=120 \\ \mathrm{n}=60 & \mathrm{n}=60 & \end{array}$

Average residual urine volume (ml) $5.0(0.0 / 18.3) 8.0(0.0 / 27.5) 7.0(0.0 / 20.3)$

Zero residual volume $\quad 26 / 43.3 \% \quad 25 / 41.7 \% \quad 51 / 42.5 \%$

Residual volume $\leqslant 50 \mathrm{ml} \quad 56 / 93.3 \% \quad 53 / 88.3 \% \quad 109 / 90.8 \%$

$\begin{array}{llll}\text { Residual volume }>50 \mathrm{ml} & 4 / 6.7 \% & 7 / 11.7 \% & 11 / 9.2 \%\end{array}$

Residual volume $>100 \mathrm{ml} \quad 2 / 3.3 \% \quad 2 / 3.3 \% \quad 4 / 3.3 \%$

The values are given as the median and the lower and upper quartiles or count and percentage, where appropriate.
Impairment Scale A) in one tetraplegic man with a C7 injury and incomplete (American Spinal Injury Association Impairment Scale B-D) in the others. Overall, SCI was complete and incomplete in 24 and 36 men, respectively.

Investigated participants evacuated their bladder a median of 5.0 times per day (LQ: 4.0, UQ: 6.0) using IC. The median residual urine volume determined by ultrasonography was $7.0 \mathrm{ml}$ (LQ: 0.0 , UQ: $20.3 \mathrm{ml}$ ). There was a median difference of $10.0 \mathrm{ml}$ (LQ: 0.0 , UQ: $28.5 \mathrm{ml}$ ) in residual urine between the first and second catheterization (Table 1). Fourteen (23.3\%) and $23(38.3 \%)$ men had emptied their bladder twice and once, respectively, without residual urine. Unsatisfactory residual urine volumes (that is, $>50 \mathrm{ml}$ ) were observed after 11 catheterizations (Table 1 ). No participant had emptied the bladder twice unsatisfactorily. No difference in the median residual urine volume and the occurrence of unsatisfactory residual volumes was observed between tetra- and paraplegic men.

The majority of participants $(n=54,90.0 \%)$ used one of three different catheters: SpeediCath (Coloplast A/S, Humlebaek, Denmark; $n=24$ ), LoFric (Wellspect HealthCare, Mölndal, Sweden; $n=18$ ) or IQ-Cath (Manfred Sauer GmbH, Lobbach, Germany; $n=12$ ). Two individuals used non-hydrophilic-coated catheters with a lubrication gel. The catheter sizes used were French gauge $14(n=44)$ and 12 $(n=16)$. The average residual urine volumes for the SpeediCath, LoFric and IQ-Cath were $3.5 \mathrm{ml}$ (LQ: 0.0, UQ: $17.3 \mathrm{ml}$ ), $14.5 \mathrm{ml}$ (LQ: 0.0, UQ: $26.8 \mathrm{ml}$ ) and $4.5 \mathrm{ml}(\mathrm{LQ}: 0.0$, UQ $12.8 \mathrm{ml} ; P=0.07)$. The maximum residual urine volumes for the SpeediCath, LoFric and IQ-Cath were $14.0 \mathrm{ml}$ (LQ: 4.8, UQ: $42.0 \mathrm{ml}$ ), $18.5 \mathrm{ml}$ (LQ: 4.0, UQ: $51.3 \mathrm{ml}$ ) and $10.0 \mathrm{ml}$ (LQ: 3.8, UQ: $23.5 \mathrm{ml} ; P=0.64$ ).

Effects of residual urine on the rate of symptomatic UTIs

A total of eight men were excluded from the analysis of the effects of residual urine volumes on the rate of symptomatic UTIs because the annual rate of UTIs could not be determined (IC $<12$ months; $n=4$ ), the age was $>65$ years $(n=3)$ or because of antibiotic treatment for $>28$ days within the previous 12 months. The data of 52 men were thus analyzed (Table 2). Thirteen men had implemented nonantibiotic UTI prophylaxis, that is, cranberry products $(n=8)$, L-methionine $(n=3)$ or both $(n=2)$. The median number of annual UTI was not significantly $(P=0.52)$ different between men with UTI prophylaxis (median 0.0, lower quartile range: 0.0; UQ: 3.0) and men without (median 0.0, lower quartile range: 0.0; UQ: 1.0). There was no significant $(P>0.94)$ difference between the median residual urine volumes and the maximal residual urine volumes of men with

Table 2 Patient characteristics, residual urine volumes and number of UTIs in men with SCI using IC

\begin{tabular}{|c|c|c|c|c|}
\hline & Men with sporadic UTIs & Men with recurrent UTIs & P-value & Total \\
\hline Count & $44 / 84.6 \%$ & $8 / 15.4 \%$ & NA & $52 / 100.0 \%$ \\
\hline Age (years) & $46.7(39.3 / 56.7)$ & $49.1(39.2 / 54.7)$ & 0.91 & $46.9(39.2 / 56.6)$ \\
\hline Duration of $\mathrm{SCl}$ (years) & $16.5(8.0 / 22.7)$ & $10.3(6.1 / 22.2)$ & 0.76 & $16.1(7.5 / 22.7)$ \\
\hline Duration of IC (years) & $11.4(6.2 / 15.4)$ & $5.4(3.7 / 8.9)$ & 0.06 & $10.3(4.6 / 13.7)$ \\
\hline UTI prophylaxis & $8 / 18.2 \%$ & $5 / 62.5 \%$ & $0.03^{a}$ & $13 / 25.0 \%$ \\
\hline Annual rate of UTIs & $0.0(0.0 / 1.0)$ & $5.0(3.8 / 7.0)$ & $<0.001$ & $0.0(0.0 / 2.0)$ \\
\hline Average residual urine volume $(\mathrm{ml})$ & $6.0(0.0 / 20.0)$ & $2.5(0.0 / 29.3)$ & 0.95 & $5.0(0.0 / 20.3)$ \\
\hline Maximum residual urine volume (ml) & $12.5(3.8 / 41.3)$ & $16.0(3.8 / 33.8)$ & 0.97 & $13.5(3.8 / 41.3)$ \\
\hline Residual volume $\leqslant 50 \mathrm{ml}$ & $80 / 90.9 \%$ & $15 / 93.8 \%$ & $0.58^{\mathrm{a}}$ & $95 / 91.3 \%$ \\
\hline Residual volume $>50 \mathrm{ml}$ & $8 / 9.1 \%$ & $1 / 6.3 \%$ & $0.58^{a}$ & $9 / 8.7 \%$ \\
\hline
\end{tabular}

Abbreviations: IC, intermittent catheterization; NA, not applicable; SCI, spinal cord injury; UTIs, urinary tract infections.

The values are given as the median and the lower and upper quartiles or count and percentage, where appropriate. Sporadic UTIs: $\leqslant 2$ UTIS per year, recurrent UTIs: $>2$ UTIs per year. a Determined by the $\chi^{2}$-test and Fisher's exact test. 
recurrent UTIs and the volumes of those with sporadic UTIs (Table 2). No significant $(P=0.58)$ difference in the frequency distribution of unsatisfactory $(>50 \mathrm{ml})$ versus satisfactory $(\leqslant 50 \mathrm{ml})$ residual volumes was detected between men with recurrent UTIs and those with sporadic UTIs (Table 2). Finally, no significant $(P=0.62)$ correlation $\left(r^{2}=0.24\right)$ between residual urine volumes and the number of annual UTI was observed.

The number of observations and sample sizes were too small for statistical analysis of the UTI rate for the different catheter groups.

\section{DISCUSSION}

In the present study, the majority (82\%) of men with NLUTD performing IC emptied their bladders without clinically relevant residual urine volumes. The median residual urine volume after IC was $7.0 \mathrm{ml}$. Sixty percent $(n=36)$ of the participants had emptied their bladder at least once without detectable residual urine. Unsatisfactory residual volumes $(>50 \mathrm{ml})^{4}$ were observed after $\sim 9 \% \quad(n=11)$ of all catheterizations $(n=120)$. There was no significant difference between the residual urine volume of men with recurrent UTIs and the volume of those with sporadic UTIs.

Our results confirm the widely held notion that IC, if applied correctly, is an efficient method for bladder evacuation, often resulting in zero residual urine. ${ }^{15}$ In the present study, no residual urine was observed after $42 \%(n=50)$ of all catheterizations $(n=120)$. Jensen et al. ${ }^{4}$ have reported no residual urine in $31 \%(n=11)$ of 36 third-party IC in 12 SCI patients. The median residual urine volume in the present study was only $7 \mathrm{ml}$. Mean residual urine volumes reported by other authors range between 9 and $40 \mathrm{ml}^{4-6}$ Notable is the wide variation in measured volumes, between 0 and $250 \mathrm{ml}$, in the present study. Nevertheless, relevant residual urine volumes $>50$ and $>100 \mathrm{ml}$ were only observed after $6 \%(n=7)$ and $3 \%(n=4)$, respectively, of all catheterizations $(n=120)$. Jensen et al. ${ }^{4}$ have reported residual urine volumes $>50$ and $>100 \mathrm{ml}$ in $19 \%(n=7)$ and $6 \%(n=2)$ of all catheterizations $(n=36)$, respectively. Wide variations in residual urine volumes after IC in some study participants were also observed by other authors. ${ }^{5}$ Interestingly, some individuals able to apply correct IC techniques do not always succeed in emptying their bladder sufficiently. However, an insufficient bladder evacuation seems to get compensated during the next catheterization.

In the present study, a higher rate of UTI was not associated with larger residual urine volumes. Therefore, the hypothesis that individuals with recurrent ( $>2$ UTIs per year) symptomatic UTIs show greater residual urine volumes after IC compared with those with sporadic ( $\leqslant 2$ UTIs per year) symptomatic UTIs was rejected. The small residual urine volumes observed presently did not predispose for infections. The risk of bacteriuria seems to increase when residual urine volumes exceed $100 \mathrm{ml} .{ }^{8}$ However, asymptomatic bacteriuria is common in the SCI population and may not be associated with the rate of symptomatic UTIs. ${ }^{16}$ According to our results, which are in accordance with the literature, residual urines volumes $>100 \mathrm{ml}$ are very rare, when correct IC techniques are applied, and thus residual urine does not seem to have an important role in the development of symptomatic UTIs in the current SCI population using IC for bladder evacuation. Even if residual urine volumes $>50$ or yet $>100 \mathrm{ml}$ occur after one catheterization, the next catheterization will most likely result in sufficient bladder evacuation. Apart from residual urine, the time span between catheterizations may affect the risk of UTIs. Anderson ${ }^{17}$ have reported a higher frequency of bacteriuria after bladder evacuation by IC every $8 \mathrm{~h}$ compared with IC every $4 \mathrm{~h}$. In the present study, IC was performed approximately five times daily, that is, the time between two catheterizations was $<5 \mathrm{~h}$.
Prophylactic measures taken by some study participants may have masked the effect of residual urine on the rate of symptomatic UTIs. However, the benefit of cranberry or L-methionine products in the prevention of UTIs in NLUTD has been refuted. ${ }^{18}$ The intake of cranberry or L-methionine products (13 participants, $25.0 \%$ ) was not associated with a lower rate of UTIs in the present study. The one individual who had been taking antibiotic treatment $>28$ days within the previous 12 months had been excluded from analysis. Besides, there is only limited and weak evidence that antibiotic prophylaxis reduces the occurrence of symptomatic UTIs in individuals using IC. ${ }^{19}$

There was no difference in the efficiency of evacuating urine from the bladder between the various catheter types used in the present study. In two other studies, residual urine volumes after IC using short- or standard-length catheters in male $^{6}$ and female ${ }^{5}$ SCI individuals have been investigated. There was no significant difference in residual urine volumes between the short- and standard-length catheters. In addition, we did not observe an effect of the catheter type on the number of annual UTIs or the rate of recurrent UTIs. The effect of hydrophilic-coated catheters on the occurrence of symptomatic UTIs in individuals performing IC has been investigated; however, conflicting results have been reported either favoring hydrophilic-coated catheters ${ }^{20}$ or showing no difference. ${ }^{21}$ The risk of symptomatic UTI with hydrophilic-coated and uncoated catheters cannot be evaluated based on the present data because only two individuals used non-hydrophilic-coated catheters.

The retrospective collection of the annual rate of UTIs pertains to the limitations of the present study. Some study participants may not have remembered the correct number of UTIs suffered during the previous 12 months. However, symptomatic UTIs have a major impact on the quality of life of affected individuals, and thus episodes of symptomatic UTIs are not easily forgotten. The variability in sensitivity, specificity, predictive values and accuracy of the specific UTI signs and symptoms listed in the National Institute on Disability and Rehabilitation Research Consensus Statement, ${ }^{7}$ which have been used in the present study, contributes to the uncertainty about the true number of annual UTIs. The reported (retrospective) number of UTIs have not been verified with urine cultures. Massa et al. ${ }^{22}$ have reported high specificities $(99 \%$ for 'fever' and 'autonomic dysreflexia'), negative predictive values (90\% for 'cloudy urine', $86 \%$ for 'foul smelling urine') and accuracies ( $83 \%$ for 'cloudy urine', $79 \%$ for 'foul smelling urine' and 'feeling sick') for the listed signs and symptoms. Sensitivities (7-67\%) and positive predictive values $(18-67 \%)$ were generally low. Thus, the true number of UTIs may have been higher than that reported in the present study. We were not able to sufficiently determine the type of UTI symptoms and signs for each study participant in order to evaluate the validity of the reported annual UTI rate. A comparison of residual urine volumes between participants with zero and participants with more than three annual UTIs may further elucidate the role of residual urine in the development of symptomatic UTIs. However, based on the small volumes observed in the present study, it is not likely that residual urine after IC represents a relevant factor in the development of symptomatic UTIs in the current SCI population. Other factors such as overdistension of the bladder, vesicoureteral reflux, high-pressure voiding, bacterial prostatitis, stones or outlet obstructions are considered to increase the risk of UTIs. ${ }^{7}$

Measuring residual urine volumes by ultrasonography is a wellestablished and accurate method. High correlations $\left(r^{2} \sim 0.9\right)$ between the bladder volumes measured by ultrasonography and the 'true' volumes have been reported. ${ }^{5}$ 
In conclusion, bladder evacuation by IC in men with NLUTD resulting from SCI is an efficient method, resulting in zero or small residual urine volumes. The small residual urine volumes generally observed after IC do not predispose for UTIs.

\section{DATA ARCHIVING}

There were no data to deposit.

\section{CONFLICT OF INTEREST}

The authors declare no conflict of interest.

1 Guttmann L, Frankel H. The value of intermittent catheterisation in the early management of traumatic paraplegia and tetraplegia. Paraplegia 1967; 4: 63-84.

2 Lapides J, Diokno AC, Silber SJ, Clean Lowe BS. intermittent self-catheterization in the treatment of urinary tract disease. J Urol 1972; 107: 458-461.

3 Stohrer M, Blok B, Castro-Diaz D, Chartier-Kastler E, Del Popolo G, Kramer G et al EAU guidelines on neurogenic lower urinary tract dysfunction. Eur Urol 2009; 56: 81-88.

4 Jensen AE, Hjeltnes N, Berstad J, Stanghelle JK. Residual urine following intermittent catheterisation in patients with spinal cord injuries. Paraplegia 1995; 33: 693-696.

5 Biering-Sorensen F, Hansen HV, Nielsen PN, Looms D. Residual urine after intermittent catheterization in females using two different catheters. Scand J Urol Nephrol 2007; 41: 341-345.

6 Domurath B, Kutzenberger J, Kurze I, Knoth HS. Clinical evaluation of a newly developed catheter (SpeediCath Compact Male) in men with spinal cord injury: residual urine and user evaluation. Spinal Cord 2011; 49: 817-821.

7 The prevention and management of urinary tract infections among people with spinal cord injuries. National Institute on Disability and Rehabilitation Research Consensus Statement. J Am Paraplegia Soc 1992; 15: 194-204.
8 Merritt JL. Residual urine volume: correlate of urinary tract infection in patients with spinal cord injury. Arch Phys Med Rehabil 1981; 62: 558-561.

9 Esclarin De, Ruz A, Garcia Leoni E, Herruzo Cabrera R. Epidemiology and risk factors for urinary tract infection in patients with spinal cord injury. J Urol 2000; 164: 1285-1289.

10 Shekelle PG, Morton SC, Clark KA, Pathak M, Vickrey BG. Systematic review of risk factors for urinary tract infection in adults with spinal cord dysfunction. J Spinal Cord Med 1999; 22: 258-272.

11 Wyndaele JJ. Complications of intermittent catheterization: their prevention and treatment. Spinal Cord 2002; 40: 536-541.

12 Pedersen JF, Bartrum RJ, Grytter C. Residual urine determination by ultrasonic scanning. Am J Roentgenol Radium Ther Nucl Med 1975; 125: 474-478.

13 Pannek J. Treatment of urinary tract infection in persons with spinal cord injury: guidelines, evidence, and clinical practice. A questionnaire-based survey and review of the literature. J Spinal Cord Med 2011; 34: 11-15.

14 Dason S, Dason JT, Kapoor A. Guidelines for the diagnosis and management of recurrent urinary tract infection in women. Can Urol Assoc J 2011; 5: 316-322.

15 Trautner BW, Darouiche RO. Prevention of urinary tract infection in patients with spinal cord injury. J Spinal Cord Med 2002; 25: 277-283.

16 Waites KB, Canupp KC, DeVivo MJ. Epidemiology and risk factors for urinary tract infection following spinal cord injury. Arch Phys Med Rehabil 1993; 74: 691-695.

17 Anderson RU. Non-sterile intermittent catheterization with antibiotic prophylaxis in the acute spinal cord injured male patient. J Urol 1980; 124: 392-394.

18 Lee BB, Haran MJ, Hunt LM, Simpson JM, Marial O, Rutkowski SB et al. Spinalinjured neuropathic bladder antisepsis (SINBA) trial. Spinal Cord 2007; 45: 542-550.

19 Niel-Weise BS, van den Broek PJ, da Silva EM, Silva LA. Urinary catheter policies for long-term bladder drainage. Cochrane Database Syst Rev 2012; 8: CD004201.

20 Li L, Ye W, Ruan H, Yang B, Zhang S. Impact of hydrophilic catheters on urinary tract infections in people with spinal cord injury: systematic review and meta-analysis of randomized controlled trials. Arch Phys Med Rehabil 2013; 94: 782-787.

21 Bermingham SL, Hodgkinson S, Wright S, Hayter E, Spinks J, Pellowe C. Intermittent self catheterisation with hydrophilic, gel reservoir, and non-coated catheters: a systematic review and cost effectiveness analysis. BMJ 2013; 346: e8639.

22 Massa LM, Hoffman JM, Cardenas DD. Validity, accuracy, and predictive value of urinary tract infection signs and symptoms in individuals with spinal cord injury on intermittent catheterization. J Spinal Cord Med 2009; 32: 568-573. 